\title{
Consensus recommendations for the use of Ambulatory Glucose Profile in clinical practice
}

\author{
STEPHAN MATTHAEI ${ }^{1}$, RAMIRO ANTUÑA DEALAIZ², EMANUELE BOSI ${ }^{3}$, MARK EVANS ${ }^{4}$, \\ NEL GEELHOED-DUIJVESTIJN5 ${ }^{5}$, MICHAEL JOUBERT 6
}

\begin{abstract}
Ambulatory Glucose Profile (AGP) is a way of displaying glucose data to reveal clinically relevant information. In a previously outlined study AGP was used to interpret glucose data recorded in patients with type 1 and 2 diabetes. These results, along with the published evidence and their own clinical experience, were discussed by an expert panel of diabetes specialists, with the aim of developing guidelines to assist clinicians in the analysis and interpretation of AGP.

This group supports the consensus view that the AGP can be an effective standard for the analysis of glucose data. Interpretation guidelines are reported in the form of an algorithm, developed to demonstrate a step-by-step approach in undertaking the analysis of an AGP report in clinical practice. These are expected to improve glycaemic control, and might help patients to better understand their glucose levels and become more involved in the management of their diabetes. Where hypoglycaemia is identified, the recommendation is to focus, as a priority, on its management. At the end of the consultation, one key message should be reinforced with the patient.

Br J Diabetes Vasc Dis 2014;14:153-157
\end{abstract}

Diabetes-Zentrum Quakenbrück, Fachabteilung für Diabetologie, Endokrinologie \& Stoffwechselerkrankungen am Christlichen

Krankenhaus, Quakenbrück, Germany

Diabeclinic, c/ Corrida, 23 - 33206 Gijón, Spain

Diabetes Research Institute, San Raffaele Hospital and San Raffaele Vita-Salute University, Via Olgettina, 60, 20132 Milan, Italy

4 Wellcome Trust/MRC Institute of Metabolic Science/Dept of Medicine, University of Cambridge, Level 4 IMS MRL, Box 289, Addenbrookes Hospital, Hills Road, Cambridge CB2 OQQ, UK

Department of Internal Medicine, Medical Centre Haaglanden, Lijnbaan 32, 2512 VA, The Hague, The Netherlands

6 Endocrinologie-Diabétologie, CHU de CAEN, Avenue de la Côte de Nacre, CS 30001, 14033 Caen, Cedex 9, France

Address for correspondence: Professor Stephan Matthaei Diabetes-Zentrum Quakenbrück, Fachabteilung für Diabetologie, Endokrinologie \& Stoffwechselerkrankungen am Christlichen

Krankenhaus, Quakenbrück, Germany

Tel: +49 (0)5431 152830

E-mail: S.Matthaei@ckq-gmbh.de

http://dx.doi.org/10.15277/bjdvd.2014.046

\author{
Abbreviations and acronyms \\ AGP Ambulatory Glucose Profile \\ CGM continuous glucose monitoring \\ IQR interquartile range
}

Key words: ambulatory glucose profile, glucose monitoring, glucose data, continuous glucose monitoring, hypoglycaemia risk assessment

\section{Introduction}

AGP is a way of displaying large amounts of glucose data to reveal clinically relevant information. ${ }^{1}$ A number of reports from the US describe how the AGP is an effective standard for the analysis of glucose data, ${ }^{2-5}$ but until now, it has not been commercially available for use in Europe (Figure 1).

This expert panel of diabetes specialists evaluated the utility of AGP in their clinical practice, and aimed to develop guidelines to assist clinicians in the analysis and interpretation of AGP data, through outlining the types of questions that should be considered.

\section{Methods}

A full description of the methods used for data collection and analysis of the AGP technique has been published by Matthaei. ${ }^{6}$ In summary, glucose data were recorded for patients with type 1 and type 2 diabetes (53 type 1 subjects, and 6 type 2) through the use of the FreeStyle Navigator CGM System (Abbott Diabetes Care, Alameda, California, USA) and analysed using an AGP Extension Software to the FreeStyle CoPilot Health Management System software (Abbott Diabetes Care, Alameda, California, USA). A working group of European diabetes specialists was involved in evaluating the technique. Evidence suggests that a minimum of 14 days of CGM data provides identification of individual glucose patterns, ${ }^{7}$ therefore it was recommended that 14 days of data were recorded for analysis. In order to establish in which "real world" clinical scenarios it would be valuable to use AGP, and how to interpret the report effectively, no protocol or criteria were set. Feedback on the technique was collated via the use of an online questionnaire, based on a total of 59 patient case studies.

The results of the analysis were reviewed by this expert panel of diabetes specialists and, in conjunction with their own experience, the recommended use of AGP was discussed and agreed. 
Figure 1. Example of Ambulatory Glucose Profile (AGP) report, for illustrative purposes, which is similar to the outputs from the FreeStyle CoPilot Health Management System software

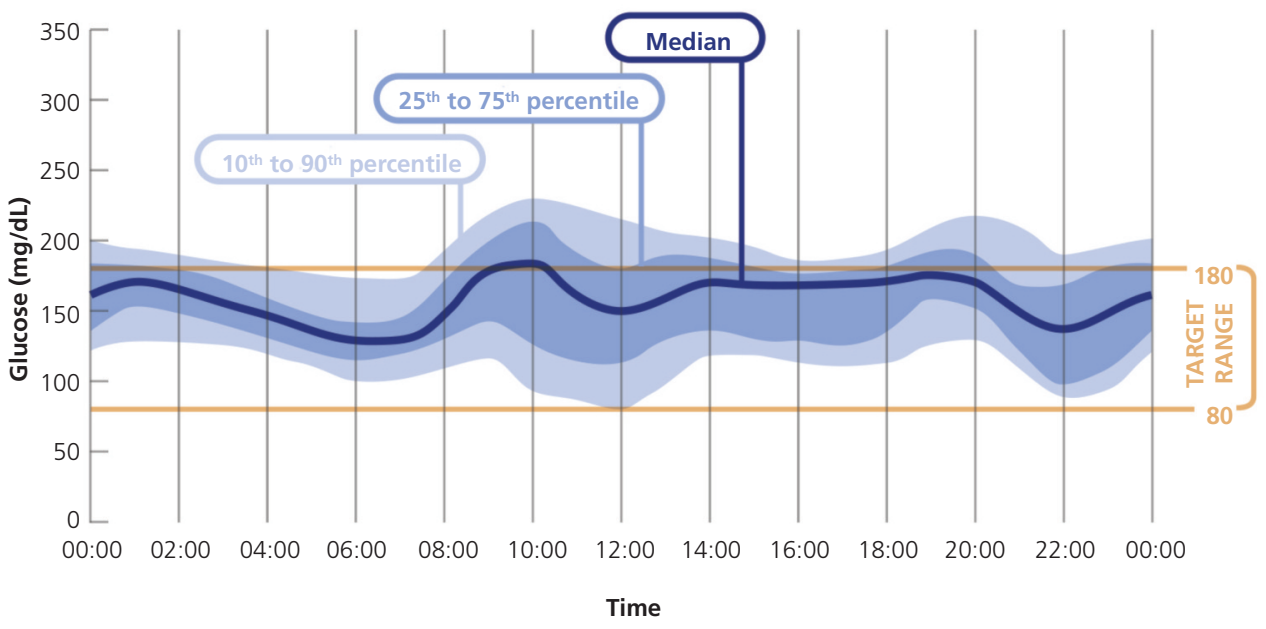

\section{Results}

Based on their clinical experience, this expert panel supports the view that AGP can be an effective standard for the analysis of glucose data. ${ }^{2-5}$ It was agreed that AGP may be valuable in the care of patients with type 1 and type 2 diabetes treated with insulin. In particular, this approach may be useful to consider in those patients with either type 1 or type 2 diabetes who have poor glycaemic control, particularly when associated with an inadequate understanding of the interaction between their condition, everyday living, and insulin use. Examples of such a patient population are those patients that struggle to manage their blood glucose, and for those who cannot identify or understand the causes of hypoglycaemic episodes. The same principles of analysis of AGP may also apply to non-insulin treated diabetes, but further work is needed to confirm this.

The information provided by the AGP may also be a useful tool for patient education. If the patient has provided supplementary data either directly into the CGM system or through the completion of a logbook, then it would be beneficial to review the AGP in conjunction with them; however, if no additional data have been provided, then it becomes essential to review the report with the patient. This creates an opportunity for discussion with the patient to analyse the patterns of glucose variations and understand better the characteristics and causes of poor control.

During the consultation, it is recommended that a step-bystep approach is undertaken to sequentially evaluate the data. This has been summarised in the algorithm in Figure 2 .

\section{Consider the patient history and quality of the data provided}

- How many days of data have been provided?

o It is generally considered that 14 continuous days of data are needed to generate an AGP report that enables optimal analysis/decision making. ${ }^{7}$
- Have carbohydrate intake/insulin doses been logged?

o It is recommended that patients input as much information on their insulin/carbohydrate intake as possible.

o If no accompanying data are provided, then review the overall glucose profile and, if this does not provide enough information, undertake a day-by-day analysis to review whether the patient exhibits a high degree of variability in their glycaemic control or profile.

2. Understand the patient's typical daily routine

- What is their insulin regimen?

o Do they use fixed insulin doses or do they vary treatment doses based on factors such as carbohydrate intake?

o Have there been any challenges maintaining their prescribed regimen?

- What is their food intake?

o How many meals and snacks do they have each day?

o What is their carbohydrate intake? Are they calculating or quantifying this accurately?

- What physical activity do they undertake?

o How frequently does the patient exercise? What impact does this have on their blood glucose?

\section{Identify hypoglycaemic patterns}

Are there periods of the day when the $10^{\text {th }}$ percentile line is in or approaching the hypoglycaemic range? If so, consider adjusting insulin dosing before this period.

- Identify individual hypoglycaemic trends: discuss possible reasons for the episodes.

- Try to ascertain whether any hypoglycaemia experienced was asymptomatic and/or required external assistance for rescue.

If hypoglycaemia is identified, the recommendation is to focus on its management, and therefore to progress to step 6 of the algorithm in Figure 2. 
Figure 2. Algorithm of the consensus recommendations for the analysis of glucose data using Ambulatory Glucose Profile (AGP)

\section{Consensus recommendations for the use of Ambulatory Glucose Profile in clinical practice}

To improve glycaemic control, it is highly recommended that the data are reviewed with the patient in order to discuss areas of concern

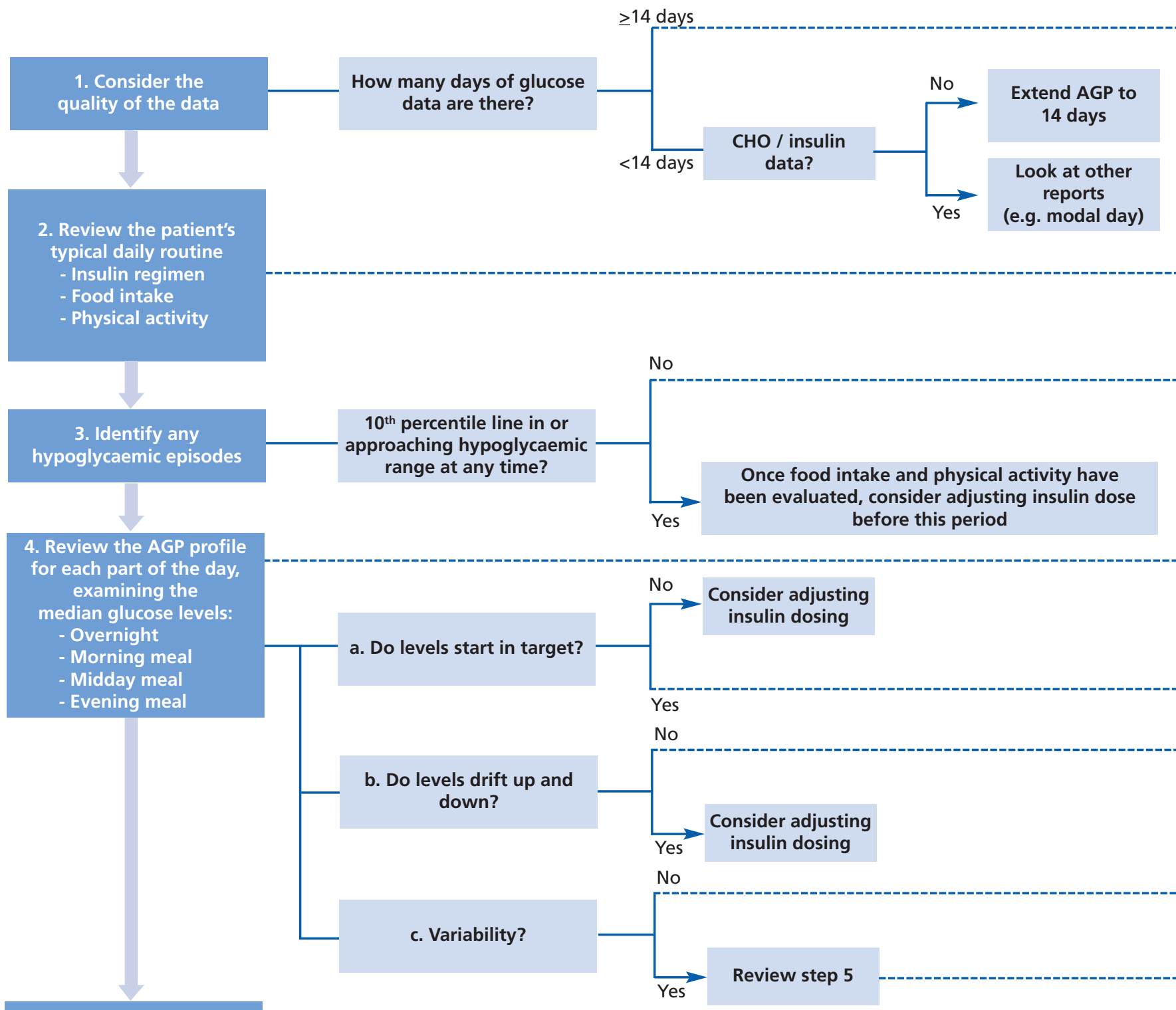

\section{Evaluate day-to-day variability}

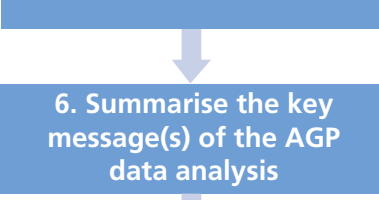

Low variability

High variability $\begin{gathered}\text { Analyse the } \\ \text { daily data }\end{gathered} \quad \begin{gathered}\text { Is the cause of variability } \\ \text { understandable? }\end{gathered}$

\section{Re-evaluate the patient}


4. Review the AGP profile for each part of the day, examining the median glucose levels:

- Overnight

- Morning meal

- Midday meal

- Evening meal

For each part of the day, looking at the AGP:

- Do glucose levels start at target?

If not, evaluate food intake, physical activity and, if indicated, consider adjusting insulin dosing.

o Do glucose levels drift up or down?
If yes, evaluate food intake, physical activity and, if indicated, consider adjusting insulin dosing.

o Is there variability?

If yes, use caution if inclined to increase insulin dosing. Review step 5

\section{Evaluate day-to-day variability}

- Are some days inconsistent with the others? If so, consider possible causes such as the impact of differing food intake, physical activity, illness, menstrual cycle, alcohol intake, insulin dose adjustments, etc.

Table 1 Example use of Ambulatory Glucose Profile (AGP) to assess a patient's glycaemic profile

\begin{tabular}{ll}
\hline & Baseline assessment \\
Clinical history & Male, 51 years old, type 2 diabetes (diagnosed 3 years ago) \\
- & Weight $93 \mathrm{~kg}$, body mass index (BMI) 32 \\
- & Received heart transplant 2 years ago, worsening of diabetes \\
& post-transplantation due to treatment with corticosteroids \\
& and immunosuppressants \\
- Glucose measurements: HbA1c $8.9 \%$, fasting blood glucose \\
readings $180-250 \mathrm{mg} / \mathrm{dL}(10-14 \mathrm{mmol} / \mathrm{l})$
\end{tabular}

\section{Week 4 assessment}

- Glucose measurements: fasting blood glucose readings $100-200 \mathrm{mg} / \mathrm{dL}(5.5-11 \mathrm{mmol} / \mathrm{l})$

AGP profile
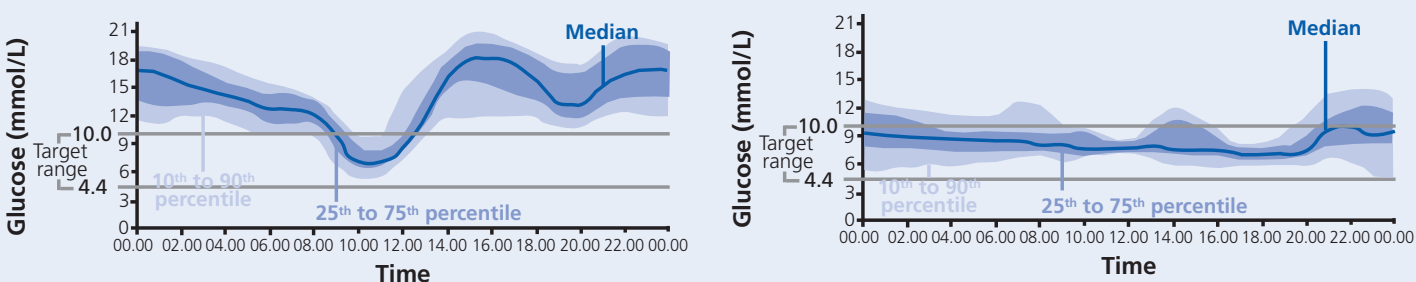

Interpretation of the AGP report

\section{Consider the quality of the data}

\section{Review the patient's typical daily routine}

\section{Identify any hypoglycaemic episodes}

4. Review the AGP profile for each part of the day, examining the median glucose levels

\section{Evaluate day-to-day variability}

\section{Reinforce one key} take-home message

\section{Re-evaluate the patient}

- A 14 day AGP is available

- Some information relating to meal timing and content has been provided by the patient

- Insulin regimen and dosage are known from the medical records

- The patient always skips breakfast but eats lunch and dinner

- Insulin treatment (initiated by transplant physician)

$$
\text { o Premix 50\% } 18 \text { units at 08:00 }
$$

- There are no hypoglycemic episodes. Dose increase of Premix $50 \%$ is limited by an evident hypoglycemic risk in the morning

- Overall hyperglycemia, especially during the night

- Major glycaemic drop in the morning due to Premix 50\% injection without meal

- Major glycaemic rise after lunch and dinner

- Low inter-day variation during night and morning demonstrated by narrow interquartile range (IQR)

- Greater variability during afternoon and evening after lunch and dinner

- Treatment was therefore adapted as follows:

o Premix insulin was discontinued

o Basal-Bolus regimen was initiated with prandial insulin before lunch and dinner

- The patient was reviewed after 4 weeks for a new Continuous Glucose Monitoring (CGM) recording
- A 14 day AGP is available

- Meal and insulin information is still available

- The patient still skips breakfast

- Insulin treatment

o Glargine: 14 units at 18:00

o Lispro: 6 units at 12:00 and 18:00

- No hypoglycemic episodes but some low glucose level in the evening

- Dramatic improvement of the overall profile

- Median glucose stable within target range throughout day (low intra-day variability), no substantial rises or falls, only moderate rise after dinner

- Low inter-day variation except after dinner, due to variable amounts of carbohydrate (patient statements)

- Dietary advice was given to the patient concerning the regularity of carbohydrate intake

- A new AGP profile could be interesting in 3-6 months 
For example, for food intake:

o Is the patient counting carbohydrates accurately?

o If patients are not counting carbohydrates, is the variability due to differing carbohydrate consumption?

- Aim to standardise carbohydrate consumption or educate the patient on carbohydrate counting.

o Is the variability due to other non-carbohydrate foods affecting glycaemic index, absorption, etc?

- Consider whether the overall picture could have been skewed by 1 or 2 anomalous days.

- Analyse the difference in the glucose levels between working and non-working days.

- In case of high variability, undertake a day-by-day analysis in consultation with the patient, to discover the underlying cause.

\section{Summary message}

At the end of the consultation, the key message(s) of the AGP data analysis should be summarised for the patient to take home to improve their glycaemic control.

\section{Re-evaluation}

Re-evaluate the patient after a period of time, checking any concerns raised in the previous consultation.

The period between evaluations should be determined by the action recommended during the first assessment. For example, for lifestyle change recommendations, the interim period will be longer than for therapy adjustments or management.

\section{Discussion}

AGP is considered to be an effective method of analysing the glucose data of patients with type 1 or type 2 diabetes. An example AGP analysis showing the recommended steps is provided (Table 1).

The expert panel members were in agreement that the AGP report is easy to interpret, and may be useful in identifying areas of concern or poor glycaemic control, including hypoglycaemia, hyperglycaemia and glycaemic variability. However, whilst the AGP report provides a useful overview of the glycaemic profile, it is also necessary to review daily data to ensure that individual excursions are not missed, for example, an individual with a severe hypoglycaemic or hyperglycaemic episode that may not be revealed on the AGP report.

In order for the report to provide most value, patients should be encouraged to input their insulin/carbohydrate intake and this should be reviewed in conjunction with the AGP report. It is important to review the report with the patient to better understand the influences and causes for poor glycaemic control. This will also provide a beneficial tool for patient education.

In summary, the method for approaching the analysis of the AGP report is to review the data quality, look for evidence of hypoglycaemia, and then divide the day into four parts for more detailed analysis and discussion with the patient.

It is critical to identify any hypoglycaemia; this can be done by analysing whether the $10^{\text {th }}$ percentile line is within or approaching the hypoglycaemic range. If hypoglycaemia is identified, then it becomes paramount to focus on its treatment and management.

Finally, in order to ensure the best outcomes, one key message,

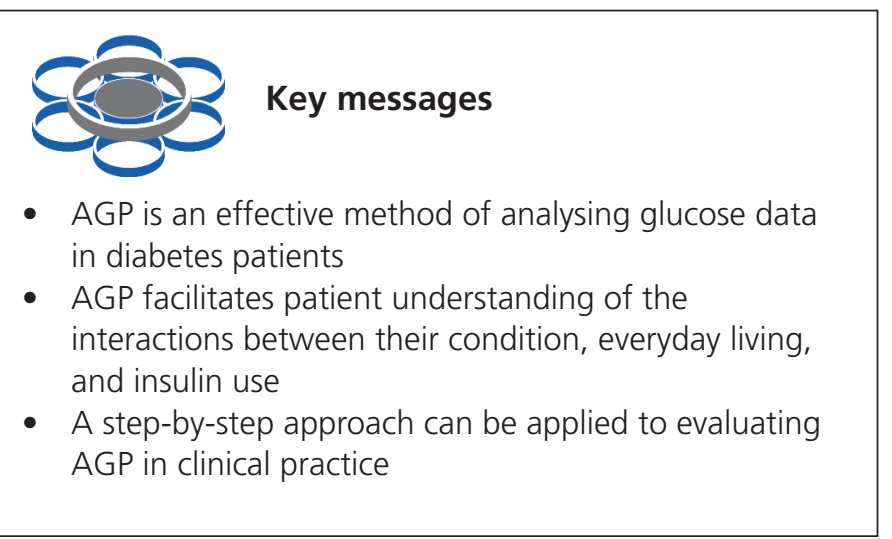

summarising how glycaemic control can be improved, should be communicated to the patient at the end of the consultation.

Conflict of interest SM has received speaker fees, and consultancy fees from Abbott Diabetes Care. ME has received speaker fees, consultancy fees, and participated in research collaborations with Abbott Diabetes Care, Medtronic, Roche, Animas, Ypsomed, NovoNordisk, Eli Lilly, CellNovo, and holds stock options in CellNovo. RA has received consultancy fees from Abbott Diabetes Care, Dexcom, and C8 Medisense, and research support from Eli Lilly, Sanofi-Aventis, and NovoNordisk. MJ has received speaker fees, consultancy fees, and research grants from Abbott Diabetes Care, Medtronic, NovoNordisk, Eli Lilly, Sanofi, Takeda, Boehringer Ingelheim, BMS, AstraZeneca, and Novartis. EB has received speaker fees, consultancy fees, and research support related to the topic of this article from Abbott Diabetes Care, Medtronic, and Roche Diabetes Care. NG-D has received speaker fees from NovoNordisk, and Abbott Diabetes Care, and consultancy fees from Abbott Diabetes Care, Roche, MSD, Medtronic, Sanofi, and Eli Lilly.

Funding sources This article was initiated and paid for by Abbott Diabetes Care, who provided FreeStyle Navigator Continuous Glucose Monitoring Systems.

Acknowledgements Medical writing support in the form of editing, referencing, journal liaison and factual accuracy checking, have been provided. However, the views expressed are those of the authors.

We thank Michael Joubert for the provision of the example case study.

\section{References}

1. Mazze RS, Strock E, Wesley D, et al. Characterizing glucose exposure for individuals with normal glucose tolerance using continuous glucose monitoring and ambulatory glucose profile analysis. Diabetes Technol Ther 2008;10:140-59. http://dx.doi.org/10.1089/dia.2007.0293

2. Bergenstal RM, Ahmann AJ, Bailey T, et al. Recommendations for standardizing glucose reporting and analysis to optimize clinical decision making in diabetes: the Ambulatory Glucose Profile (AGP). Diabetes Technol Ther 2013;15:198-211. http://dx.doi.org/10.1089/ dia.2013.0051

3. Serrano K. FDA supports standardized reporting and analysis on CGM devices. Diabetes Technol Ther 2013;15:348. http://dx.doi.org/10.1089/dia.2013.8313

4. Kaufman FR. It's time for a standard glucose report from CGM. Diabetes Technol Ther 2013;15:197. http://dx.doi.org/10.1089/dia.2013.0061

5. Kowalski A, Dutta S. It's time to move from the A1c to better metrics for diabetes control. Diabetes Technol Ther 2013;15:194-6. http://dx.doi.org/10.1089/dia.2013.0060

6. Matthaei, S. Assessing the value of ambulatory glucose profile (AGP) in clinical practice. Br J Diabetes Vasc Dis 2014:4:148-52. http://dx.doi.org/10.15277/bjdvd.2014.045

7. Dunn TC, Crouther N. Assessment of the variance of the ambulatory glucose profile over 3 to 20 days of continuous glucose monitoring. Diabetologia 2010;53 (Suppl. 1):S421. 